\title{
Clinical diagnosis of oral erosive lichen planus by direct oral microscopy
}

\author{
Barbara Drogoszewska ${ }^{1,2}$, Piotr Chomik ${ }^{1,2}$, Adam Polcyn $^{2}$, Adam Michcik $^{1,2}$ \\ ${ }^{1}$ Chair and Department of Oral and Maxillofacial Surgery, Medical University of Gdansk, Poland \\ Head of Department: Prof. Adam Włodarkiewicz MD, DMD, PhD \\ ${ }^{2}$ Department of Otolaryngology, Ward of Maxillofacial Surgery, University Clinical Center, Gdansk, Poland \\ Head of Department: Prof. Adam Włodarkiewicz MD, DMD, PhD
}

Postep Derm Alergol 2014; XXXI, 4: 222-228

DOI: $10.5114 /$ pdia.2014.40926

\begin{abstract}
Introduction: Direct oral microscopy is a novel, non-invasive diagnostic technique that aids clinical examination of the oral cavity. The basic principles of this method derive from colposcopy and dermoscopy. The principle is to reveal precancerous lesions of oral mucosae in their subclinical phase in order to begin their treatment as early as possible and prevent malignant transformation. Oral lichen planus (OLP) is an autoimmune, inflammatory, chronic disease affecting oral mucous membranes. Buccal mucosae are most often affected.

Aim: To describe the in vivo picture of erosive OLP in direct oral microscopy in terms of the pattern and density of subepithelial blood vessels, surface texture, color, transparency and borders of the lesions. The study also demonstrates the utility of the method in the selection of the most appropriate biopsy site.

Material and methods: A total of 30 patients with erosive OLP were examined. Clinical examination of the oral cavity with the naked eye was performed, followed by direct oral microscopy. The most appropriate biopsy sites based on both examinations were chosen for every individual and biopsies were taken for histopathological evaluation. Results: Biopsies obtained based on direct oral microscopy revealed dysplasia in 16 patients (53.3\%). Biopsies obtained based on clinical examination with the naked eye revealed dysplasia in 3 cases (10\%).

Conclusions: Direct oral microscopy makes it possible to obtain a repeated picture of erosive OLP and constitutes an alternative to the clinical examination with the naked eye in election of the most appropriate biopsy site. Thus, introduction of the most accurate and early therapy is possible.
\end{abstract}

Key words: direct microscopy, oral cavity, colposcopy, lichen planus, diagnosis.

\section{Introduction}

Direct oral microscopy is a novel non-invasive diagnostic technique so far utilized sporadically that aids clinical examination of the oral cavity. The basic principles of this method derive from colposcopy, a common diagnostic tool in gynecology and dermoscopy that is utilized in the clinical evaluation of skin lesions. In this technique the oral mucosae are examined using a microscope at multiple magnifications and features such as subepithelial mucosal vessels, surface pattern, color tone, transparency, and the exact lines of demarcation of mucosal lesions are examined. Application of a green filter to the light source provides better contrast between subepithelial vessels and the surrounding tissues, which facilitates their examination [1].
Oral lichen planus (OLP) is an autoimmune, inflammatory, chronic disease affecting oral mucous membranes. The pathogenesis of OLP is based on a T lymphocyte-dependent immunological reaction [2]. The incidence of oral mucosae involvement in the course of the disease is estimated to be 0.3-2.5\% [3]. Clinically, six types of mucosal lesions can be identified: network, papulous, plaque-like, erosive, atrophic and bullous. Individual patients may be affected by more than one type of lesion. Malignant transformation most often involves erosive, atrophic and plaque-like lesions, but is also possible in the remaining types. The rate of malignant transformation in OLP has been estimated to be approximately $1 \%$ in all cases [3]. To date, the exact mechanism of malignant transformation in OLP has not been described. One hypothesis is that altered oral mucosae are more susceptible to carcinogens. On the other hand, cases of malig-

Address for correspondence: Piotr Chomik DMD, PhD, Ward of Maxillofacial Surgery, University Clinical Center, 17 Smoluchowskiego St, 80-214 Gdansk, phone: +48 5834930 90, fax: +48 5834931 00, e-mail: piotr.chomik@gumed.edu.pl, wlodarkiewicz@gumed.edu.pl Received: 14.05.2013, accepted: 27.08.2013. 
nant transformation within lichen planus without the influence of carcinogens have been observed [3]. This indicates the need for novel diagnostic methods capable of capturing cases of lichen planus that are particularly prone to malignant transformation.

A common feature of precancerous mucosal lesions is the fact that they may contain both typical and atypical cells, as well as cells that have already begun the process of malignant transformation [4]. Thus, the histopathological result of the biopsy in such lesions may depend both on the region biopsied within the lesion and the pathologist's experience. This indicates that the histopathological result contains a significant subjective component, which is crucial for further therapeutic decisions. Thus, objective diagnosis of precancerous lesions, as well as the selection of the most representative biopsy sites to obtain the most altered tissue material, are of vital importance.

\section{Aim}

The aim of this study was to describe the in vivo picture of erosive OLP in direct oral microscopy in terms of the pattern and density of subepithelial blood vessels, surface texture, color, transparency and borders of the lesions. The study also demonstrates the utility of direct oral microscopy in the selection of the most appropriate biopsy site in erosive OLP.

\section{Material and methods}

Adult patients, aged 24-81 (mean age: 52.5 ) years old referred to the Outpatient Office of Maxillofacial Surgery and Ward of Maxillofacial Surgery in the Department of Otolaryngology, University Clinical Centre in Gdansk, Poland were included in the study. A total of 30 patients ( 5 males, 25 females) presenting lesions typical of erosive OLP were enrolled. For every individual the clinical diagnosis was confirmed histopathologically. Information about general health status, smoking habits, alcohol consumption and other addictions was collected for every patient. Interestingly, $60 \%(n=18)$ of patients had previously smoked more than 20 cigarettes daily, and at the time of examination, $36.7 \%(n=11)$ of patients admitted to smoking the same number despite the presence of precancerous oral lesions. At the time of examination, $26.7 \%(n=8)$ of patients used removable dental prostheses. Prior to the study, every patient gave written consent to the examination and biopsy after having been informed about the procedure and aim of the study. The study design was accepted by the Institutional Review Board of the Medical University in Gdańsk (NKEBN/137/2011).

Patients were first thoroughly examined under a halogen lamp with the naked eye and the most representative site for biopsy was chosen. Direct oral microscopy was subsequently performed in a darkened room and, based on colposcopic features (subepithelial mucosal vessels, surface pattern, color tone, transparency and borders of the lesion), the most suitable site for biopsy was indicated. Two biopsy specimens were collected: the first from the site chosen during clinical examination with the naked eye and the second from the site indicated by direct oral microscopy. In cases of overlap, the second specimen was collected from a region randomly chosen within the lesion based on clinical examination. All biopsies were performed under local anesthesia with $2 \%$ lignocaine.

The control group consisted of 30 patients (16 males, 14 females), aged 37-61 (mean age: 49) years old, who presented clinically unaltered oral mucosae treated due to maxillofacial trauma and/or cystic tumors of the maxillofacial skeleton.

Direct oral microscopy was performed using a Leisegang colposcope, model BG/LED Y/C type 3ML (Leisegang, Germany). Photographs were taken with a Canon EOS 500D digital camera equipped with a Canon Macro 100 mm lens (Canon, Japan). An Apple iMac computer with Mac OS X software (Apple, CA, USA) was utilized for the analysis, processing and archiving of pictures.

Differences in the intensity of particular features observed in the pictures were estimated according to an established numeric scale, where "O" meant the absence of the feature, "1" meant slight/weak presence of the feature, " 2 " indicated moderate intensity of the feature and " 3 " indicated severe intensity of the described feature.

\section{Statistical analysis}

Differences between particular groups of features or alterations visible in direct oral microscopy compared to unaltered oral mucosae were estimated using the Mann-Whitney $U$ test. The correlation between the most severely expressed feature in direct oral microscopy and the intensity of the remaining features was estimated using Spearman's range correlation ratio test. Differences in the incidence of dysplasia in specimens collected based on direct oral microscopy and clinical examination were estimated using the test for the two indicators of structure. A $p$ value less than 0.05 was considered statistically significant. Statistical analysis was conducted using Statistica 10 PL software (StatSoft, Poland).

\section{Results}

Thirty patients with erosive OLP were examined using direct oral microscopy. They presented diffuse lesions on the buccal mucosae, in which subepithelial capillaries were not visible, even after application of a green filter to the white light source (Figure 1). It should be noted that in no case was the site for biopsy chosen via direct oral microscopy the same as the region biopsied based on clinical examination with the naked eye. 


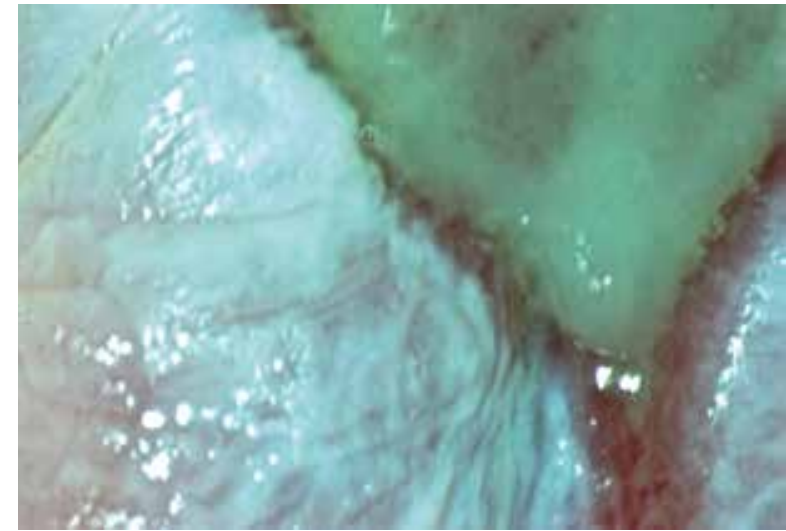

Figure 1. Erosive OLP of buccal mucosae. The subepithelial capillaries were not visible even after application of a green filter (magnification 30x)

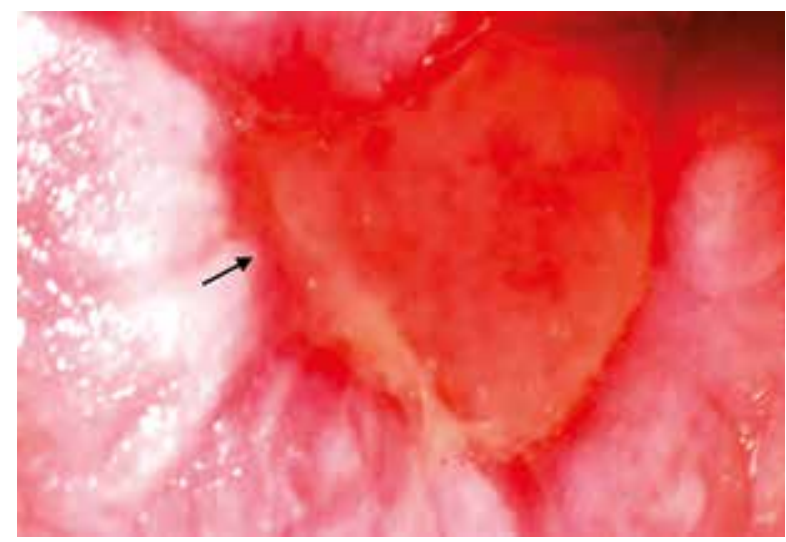

Figure 3. Erosive OLP. Light red color of erosion. Typical, intensive red edge (arrow), demarcating erosion from white, hyperkeratotic lesions (magnification 30x)

The invisibility of subepithelial capillaries within the lesions was undoubtedly due to the surface pattern of OLP. The coexistence of planar-elevated, whitened, dull, rough, slightly folded and hyperkeratotic leukoplakia-type lesions was observed, together with furrows and cracks, where the squamous epithelium was considerably thickened. Such lesions were directly adjacent to erosions, namely lesions devoid of thick squamous epithelium with a glossy, smooth and moist surface. The bottom of these erosions was located slightly below the surface of neighboring white, hyperkeratotic lesions (Figure 2).

The typical bi-color of erosive OLP was evident. The whitened and dull epithelium distinctive of leukoplakia was observed within hyperkeratotic lesions, whereas erosions were light to intense red with a typical sharp demarcation line between white lesions and erosions (Figure 3).

The thickened layer of squamous epithelium within the white lesions contributed to reduced transparency, which resulted in the inability to observe subepithelial structures. Thinner, regenerating epithelium on the bor-

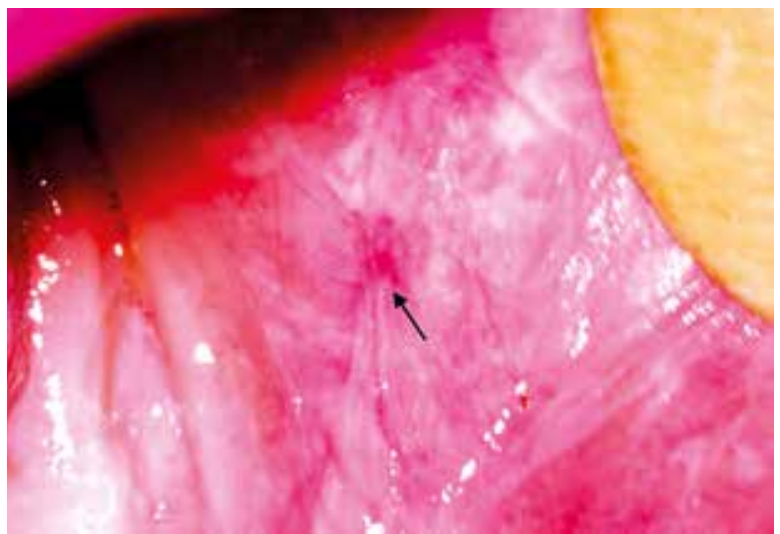

Figure 2. Erosive OLP of buccal mucosae. White, hyperkeratotic lesions with erosion (arrow) devoid of thick, hyperkeratotic epithelium (magnification 15x)

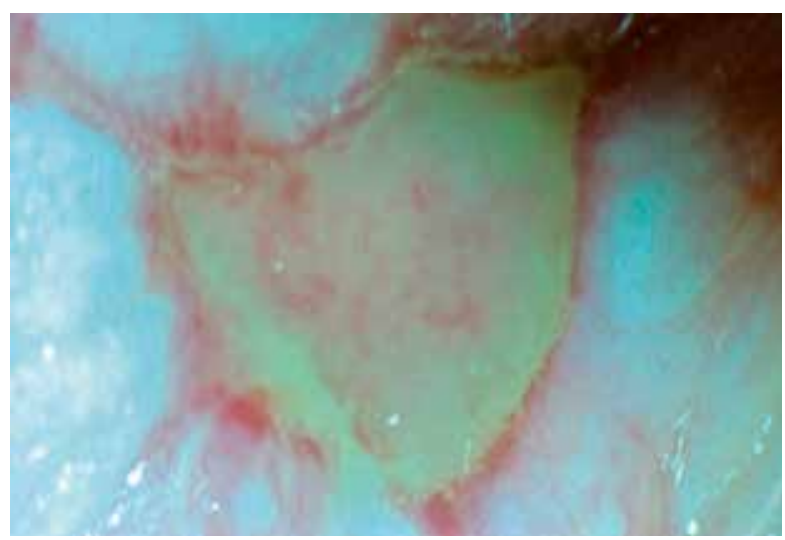

Figure 4. Erosive OLP. Subepithelial structures were not visible within white lesions and erosions due to disturbed architecture of thin epithelium (magnification 15×)

ders of erosions revealed a disturbed architecture, which again limited the visibility of subepithelial structures, due to light dispersion (Figure 4).

Cases of erosive OLP in this study mostly presented diffuse lesions on the buccal mucosae. Twelve (40\%) patients had extensive bilateral lesions. The remaining $18(60 \%)$ individuals had more limited unilateral lesions. Unclear borders between whitened lesions and unaltered oral mucosae, as well as a sharp intensive red demarcation line between whitened areas and erosions were apparent (Figure 5).

Statistical analysis of the intensity of particular estimation parameters (subepithelial capillaries, surface pattern, color tone, transparency, lines of demarcation) revealed significant differences between erosive OLP in direct oral microscopy compared to the established pattern of healthy oral mucosae $(p<0.05$ for every estimation parameter). Combination of each parameter's intensity within erosive OLP seen in direct oral microscopy compared to unaltered oral mucosae is presented in Table 1. 


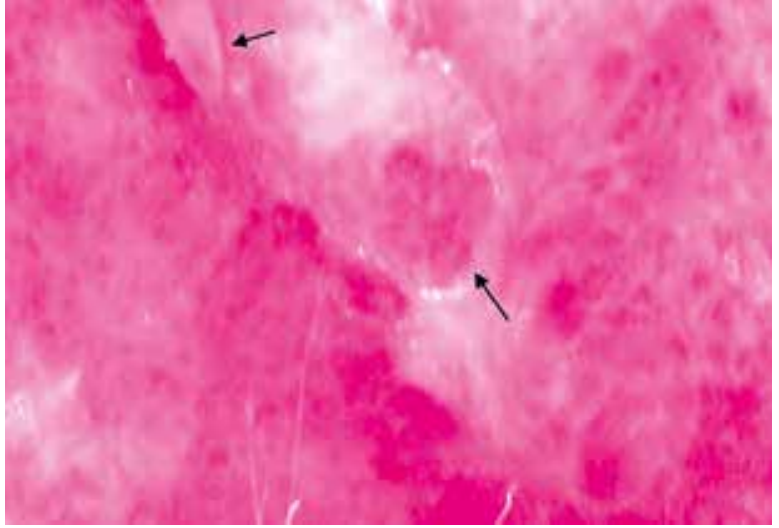

Figure 5. Erosive OLP. Sharp demarcation of red erosions (arrows) from surrounding white hyperkeratotic lesions, which present unclear borders with healthy oral mucosae (magnification 30x)

The histopathological results of biopsies obtained from areas with the highest alterations in intensity compared to healthy mucosae in direct oral microscopy revealed low-grade (mild) dysplasia in 9 cases (30\%) and moderate dysplasia in 7 patients (23.3\%) treated due to erosive OLP. In other words, dysplasia was found in 16 individuals, equating to $53.3 \%$ of patients with erosive OLP (Table 2).

The histopathological results of biopsies obtained based on clinical examination with the naked eye revealed low-grade dysplasia in only 2 cases (6.7\%) of erosive OLP and moderate dysplasia in 1 patient (3.3\%). Thus, only $10 \%$ of patients treated due to erosive OLP showed features of dysplasia in specimens obtained after examination with the naked eye. Analysis of the detection frequency of dysplasia in specimens obtained based on direct oral microscopy compared to clinical examination with the naked eye in patients with erosive OLP revealed a statistically higher rate of detection of dysplasia in biopsies based on the former method $(p=0.0003)$. A comparison of the frequency of dysplasia in specimens obtained after direct oral microscopy and clinical examination with the naked eye is presented in Table 3.

Assessment of subepithelial blood vessels proved to be the most reliable prognostic parameter of direct oral microscopy in terms of presence of dysplasia.

Subepithelial blood vessels were not visible within the lesions of the 30 patients examined due to erosive OLP; however direct oral microscopy revealed subepithelial capillaries around the lesions, in close proximity to their borders, in 9 cases (30\%). In 6 individuals (20\%) they were identified as hairpin capillaries (Figure 6) according to Gynther et al. [1], whereas in the remaining 3 patients (10\%) punctuation capillaries were observed, as described by Gynther et al. [1] (Figure 7). In agreement
Table 1. Combination of lesion intensity within erosive OLP seen in direct oral microscopy compared to unaltered oral mucosae

\begin{tabular}{lcc}
\hline Feature & $\begin{array}{c}\text { Unaltered } \\
\text { oral mucosae }\end{array}$ & Erosive OLP \\
\hline $\begin{array}{l}\text { Image of subepithelial } \\
\text { capillaries }\end{array}$ & $1 / 2$ & $\begin{array}{c}0 \\
(p=0.000000000003)\end{array}$ \\
\hline $\begin{array}{l}\text { Image of pathologic } \\
\text { vessels around the lesion }\end{array}$ & 0 & $\begin{array}{c}1 \\
(p=0.00000000003)\end{array}$ \\
\hline $\begin{array}{l}\text { Moist, smooth, glossy } \\
\text { surface }\end{array}$ & $1 / 2$ & $\begin{array}{c}1 / 0 \\
(p=0.000000628489)\end{array}$ \\
\hline $\begin{array}{l}\text { Planar (flat) surface } \\
\text { Rosy colour }\end{array}$ & 1 & $\begin{array}{c}0 / 1 \\
(p=0.001952845490)\end{array}$ \\
\hline White colour & $1 / 2$ & $\begin{array}{c}0 \\
(p=0.00000000003)\end{array}$ \\
\hline Red colour & 0 & $(p=0.00000000003)$ \\
\hline Transparency & 0 & $\begin{array}{c}2 / 3 \\
(p=0.00000000003)\end{array}$ \\
\hline Borders of the lesion & 0 & $\begin{array}{c}0 \\
(p=0.00000000003)\end{array}$ \\
\hline
\end{tabular}

0 - lack of feature, 1 - mild intensity of feature, 2-moderate intensity of feature, 3 - severe intensity of feature. Differences statistically significant when $p<0.05$

Table 2. Combination of histopathological results of biopsies from erosive OLP based on direct oral microscopy

\begin{tabular}{lc}
\hline Degree of dysplasia & Erosive OLP $(\boldsymbol{N}=30)$ \\
\hline Mild dysplasia & $9(30 \%)$ \\
\hline Moderate dysplasia & $7(23.3 \%)$ \\
\hline Total & $16(53.3 \%)$ \\
\hline
\end{tabular}

Table 3. Comparison of frequency of epithelial dysplasia in biopsies from erosive OLP obtained in direct oral microscopy and clinical examination with the naked eye

\begin{tabular}{lcc}
\hline $\begin{array}{l}\text { Degree of } \\
\text { dysplasia }\end{array}$ & $\begin{array}{c}\text { Direct oral microscopy } \\
(N=30)\end{array}$ & $\begin{array}{c}\text { Naked eye } \\
(N=30)\end{array}$ \\
\hline Mild dysplasia & $9(30 \%)$ & $2(6.7 \%)$ \\
\hline Moderate dysplasia & $7(23.3 \%)$ & $1(3.3 \%)$ \\
\hline Total & $\begin{array}{c}16(53.3 \%) \\
(p=0.0003)\end{array}$ & $\begin{array}{c}3(10 \%) \\
(p=0.0003)\end{array}$ \\
\hline
\end{tabular}

Differences statistically significant when $p<0.05$

with colposcopic criteria, hairpin capillaries were considered normal, whereas punctuation capillaries constituted a reason to increase oncologic vigilance within the examined region. Correlation ratio analysis revealed that the less visible subepithelial capillaries around the lesions, 


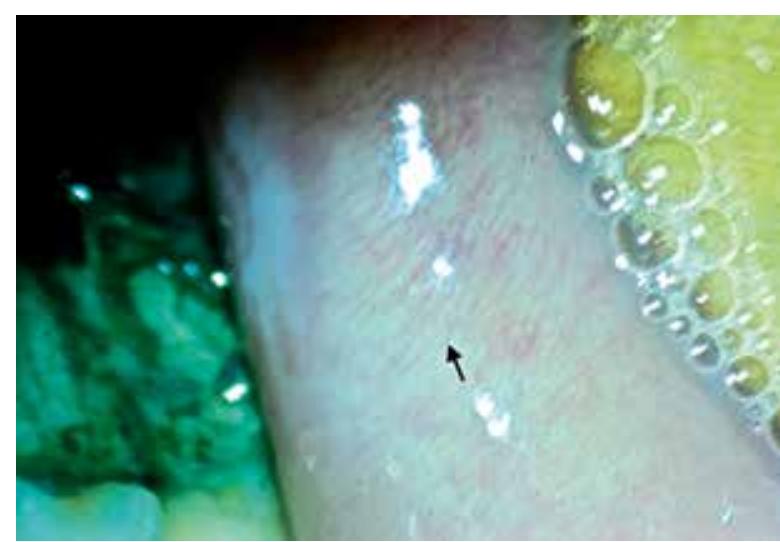

Figure 6. Numerous, thin, elongated subepithelial hairpin capillaries (arrow) visible under the green filter in buccal mucosae of a patient with erosive OLP (magnification 30x)

the more alterations within erosive OLP were observed in direct oral microscopy $(r=-0.94, p<0.05)$.

\section{Discussion}

In vivo imaging techniques are increasingly popular in modern medicine. The constant progress in biomedical engineering technologies is generating more accurate results, which in turn improves the clinical evaluation of patients and histopathological specimen interpretation. To date, these methods have been successfully applied in specialties such as gynecology and dermatology. Colposcopic examination together with dye tests is routinely performed to improve clinical gynecological evaluation, and has been recognized as crucial in cases of incorrect or unsatisfactory cytology of the uterine cervix [5]. The long-term application of colposcopy in a wide range of gynecologic diagnostic tools, as well as the availability of increasingly more sophisticated equipment, has contributed to the relatively high sensitivity and specificity of this diagnostic method [6].

Dermatoscopy (epiluminescence microscopy - ELM) has achieved similar significance in dermatology. The particular effectiveness of this method was demonstrated in the diagnosis of melanocytic lesions, skin cancers and melanomas, as well as in clinical differentiation between benign skin lesions and those suspected of malignant transformation [7]. The use of dermatoscopy to diagnose oral mucosal melanocytic lesions has been reported [8-11]. This method was shown to be particularly effective for estimating the borders of pathologic lesions [12].

Technological progress in recent years has resulted in the development of sophisticated tools dedicated to in vivo diagnostic procedures of the oral cavity at high magnification. Reports describing in vivo imaging of the oral mucosa in search of potentially malignant lesions are still rare, however. For this reason, interpretation of

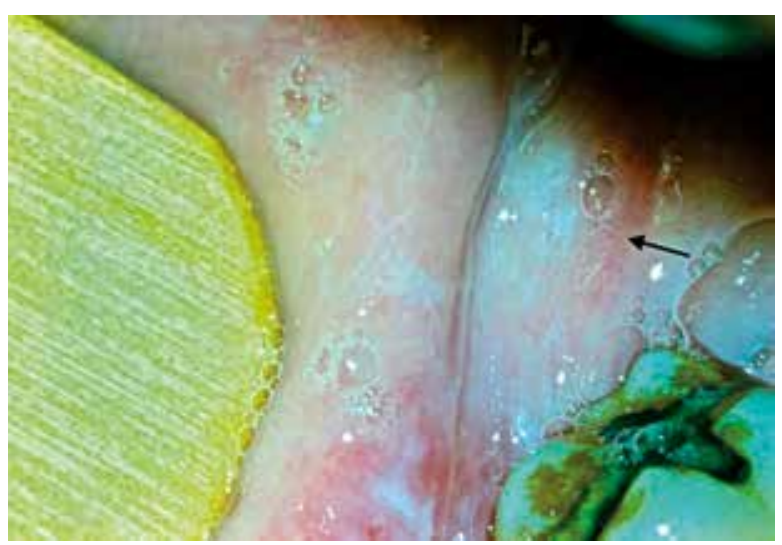

Figure 7. The arrow indicates subtle subepithelial punctuation capillaries seen under the green filter in oral mucosae surrounding lesions in a patient with erosive OLP (magnification $15 x$ )

the results obtained in this work was based mainly on gynecological and dermatological literature, because the state of medical knowledge concerning in vivo tissue imaging in these disciplines enables reliable conclusions.

In this study, the oral mucosa in patients with erosive OLP was examined via direct oral microscopy. The wide extent of the lesions, as well as the frequent bilateral involvement of the buccal mucosae were apparent. Morphologically the lesions appeared as homogenous leukoplakia-type alterations with mucosal erosions within these whitish regions. Subepithelial blood vessels were not visible within the affected areas, either under white light, or after adding a green filter. In gynecology erosive LP mostly affects the vulvar and vaginal mucosae. Lesions, similarly to those in the oral cavity, appear as diffuse, multiple erosions, and may be surrounded by white, hyperkeratotic epithelium [13]. Subepithelial capillaries are not visible on colposcopic examination, either in white light or under a green filter [14].

The mucosal surface in erosive OLP was non-homogenous. Hyperkeratotic, planar-elevated, folded, dull and dry areas were dominant. Erosions were disseminated among these regions and their surface was smooth, plane, glossy and located slightly below the surrounding white lesions. Erosions were devoid of a thick layer of squamous epithelium. The surface of vulvar and vaginal erosive LP in colposcopic examination is characterized by the presence of numerous plane and glossy erosions with a smooth bottom [15], and they are more numerous than in the oral cavity. However, similarly to erosive OLP, they are surrounded by thick, hyperkeratotic squamous epithelium [13-15].

The bi-color nature of lesions in erosive OLP was typical. The thickened, hyperkeratotic epithelium was white, which was typical of leukoplakia, whereas erosions were light to intense red. Colposcopic pictures of vulvar and vaginal erosive LP revealed similar bi-coloration, where 
erosions were intense red, but the surrounding thick and keratinized epithelium was uniformly white, similar to homogenous leukoplakia $[14,16]$.

The hyperkeratotic white epithelium of erosive OLP overlaying the buccal mucosae was totally non-transparent. However, the thin squamous epithelium at the edge of the erosions revealed disturbed polarity, which resulted in intense light dispersion and prevented the observation of subepithelial structures; nevertheless, transparency itself was preserved. In comparison with vulvar and vaginal erosive LP, the epithelium was opaque in hyperkeratotic lesions surrounding erosions, whereas within healing erosions a thin layer of regenerating epithelium hampered clear observation of subepithelial structures because of its disturbed architecture, although transparency itself remained unaltered [14].

Direct oral microscopy of erosive OLP revealed the considerable spread of the lesions, which were localized mainly on the buccal mucosae either uni- or bilaterally. White, hyperkeratotic lesions presented unclear borders with healthy oral mucosae. On the other hand, erosions located within these white lesions had clear borders visible as an intense red line. Analysis of colpophotograms of vulvar and vaginal erosive LP showed that, similarly to the oral cavity, red erosions were sharply demarcated from surrounding hyperkeratotic epithelium, which in turn gradually merged with healthy mucosae [13-16]. Sharp demarcation between erosions and the surrounding mucosae of the uterine cervix was also evident in colposcopic examination [15]. Moreover, a study concerning dermoscopic examination of lesions on lower genital tract mucosae proved that erosions were sharply demarcated from the surrounding unaltered epithelium [17].

A statistically significant difference in the intensity of particular estimation parameters between erosive OLP and healthy oral mucosa was found. In order to limit the influence of the examiner's subjective feelings on image interpretation, further studies on the effectiveness of direct oral microscopy in oral mucosae diagnostics are required.

Histopathologic analysis of biopsies obtained from erosive OLP based on direct oral microscopy revealed features of epithelial dysplasia in $53.3 \%$ of cases. According to the literature, such a rate is surprisingly high as various authors have reported that the rate of malignant transformation of OLP oscillates between $0.5 \%$ and $12.5 \%$ over time and did not exceed $1 \%$ in 5 -year observations [18-22]. Researchers have been debating whether lesions with the clinical appearance of OLP with features of epithelial dysplasia should be classified histopathologically as LP [20]. The term "lichenoid dysplasia" has been proposed as an alternative $[18,20]$. In this context the notion of the presence of dysplasia in LP lesions that have already begun the process of malignant transformation is reasonable. Thus, the statement that lesions with proven epithelial dysplasia should not be classified as LP seems to be unjustified [20]. Such a strategy may lead to underestimation of the rate of malignant transformation in LP. Thus, the use of direct oral microscopy in the selection of the most appropriate biopsy site in erosive OLP seems reasonable. Given that dysplasia may be associated with OLP cases that have begun the process of malignant transformation, such patients demand a very precise treatment plan, as well as strict periodic clinical and histopathological check-ups. It should also be noted that the low rates of dysplasia and malignant transformation in OLP mentioned above were established based on biopsies obtained from sites selected with the naked eye, without techniques for improving in vivo tissue examination. The considerably higher and statistically significant epithelial dysplasia rate in biopsies from erosive OLP obtained in direct oral microscopy compared to clinical examination with the naked eye in this study may indirectly indicate the effectiveness of the method in the selection of the most appropriate biopsy site. When comparing the dysplasia rate in biopsies obtained from direct oral microscopy (53.3\%) with the dysplasia rate in biopsies obtained from clinical examination with the naked eye (10\%), the latter also seems high, although comparable with data from the literature [22]. Such a result indirectly supports the idea that exclusion of an LP diagnosis in lesions that show dysplasia is not fully justified and that the real dysplasia rate may be higher in this entity. Further studies on numerous groups of patients are needed to fully verify this concept.

Assessment of the diagnostic value of particular estimation parameters adapted from colposcopy to direct microscopy of oral mucosa revealed that estimation of subepithelial vessels was the most reliable. Direct oral microscopic examination of patients with erosive OLP revealed subepithelial capillaries around the lesions in $30 \%$ of cases, whereas $10 \%$ revealed abnormal punctuation vessels. There was a statistically significant negative correlation between the visibility of vessels around the lesions and the severity of alterations seen in direct oral microscopy. In the face of controversies regarding the diagnosis of OLP and estimation of its malignant transformation rate, direct oral microscopy may provide a new direction for research, which will hopefully result in verification of the real malignant potential of the disease. Such observations are equal with analyses of colposcopic and dermoscopic images of vulvar and vaginal mucosa, where in most cases, lesions of erosive LP were not associated with surrounding subepithelial vessels [13-17].

Analyses of particular images of erosive OLP in direct oral microscopy, as well as the comparison of histopathological results in this study, led to the conclusion that direct oral microscopy may constitute an alternative to clinical examination with the naked eye in the selection of the most appropriate biopsy site in order to achieve the most reliable histopathological results. This in turn should enable the initiation of appropriate treatment as quickly as possible. 


\section{Conclusions}

The adaptation of in vivo tissue imaging techniques that are commonly utilized in gynecology and dermatology to diagnose oral mucosae constitutes a novel research field that aims to improve the diagnostic procedures of oral precancers and cancers and improve their treatment outcomes. The availability of precise diagnostic equipment, together with a growing social awareness concerning oral cavity health, raises the hope that these goals may be achieved in the near future.

\section{Conflict of interest}

There is no conflict of interest.

\section{References}

1. Gynther GW, Rozell B, Heimdahl A. Direct oral microscopy and its value in diagnosing mucosal lesions. A pilot study. Oral Surg Oral Med Oral Pathol Oral Radiol Endod 2000; 90: 164-70.

2. Bombeccari GP, Guzzi G, Tettamanti M, et al. Oral lichen planus and malignant transformation: a longitudinal cohort study. Oral Surg Oral Med Oral Pathol Oral Radiol Endod 2011; 112: 328-34.

3. Włodarkiewicz A (ed.). Dermatochirurgia [Polish]. Cornetis, Wrocław 2009.

4. Scully C, Petti S. Overview of cancer for the healthcare team: aetiopathogenesis and early diagnosis. Oral Oncol 2010; 46: 402-6.

5. Nastałek M, Wojas-Pelc A. The assessment of microcirculation architecture in keloids and hypertrophic scars: videocapillaroscopy. Postep Derm Alergol 2011; 28: 423-7.

6. Florek M, Wojtuń S, Gil J. New methods of identification and screening of Barrett's esophagus [Polish]. Pol Merk Lek 2009; 26: 524-6.

7. Florek M, Wojtuń S, Gil J. New possibilities for detection of adenomas of the colon [Polish]. Pol Merk Lek 2009; 26: 562-4.

8. Xiumei W, Wenjing S, Jing B, et al. Growth inhibition induced by transforming growth factor beta- 1 in human oral squamous cell carcinoma. Mol Biol Rep 2009; 36: 861-9.

9. Strycharz M, Polz-Dacewicz M, Gołąbek W, et al. The epidemiologic analysis of 254 oral cancer cases from the Lublin region. Annales Universitatis Mariae Curie-Skłodowska, Lublin 2006; LXI, N 2, 114, Sectio D; 655-9.

10. Rigual NR, Wiseman SM. Neck dissection: current concepts and future directions. Surg Oncol Clin N Am 2004; 13: 151-66.

11. Poswillo D. Evaluation, surveillance and treatment of panoral leukoplakia. J Max Fac Surg 1975; 3: 205-11.

12. Girard KR, Strahs GR, Chung B. Panoral verrucous dysplasia. Report of a case treated with split-thickness skin graft. Oral Surg Oral Med Oral Pathol 1980; 50: 124-6.

13. Pipkin C. Erosive diseases of the vulva. Dermatol Clin 2010; 28: 737-51.

14. Duong TH, Flowers LC. Vulvo-vaginal cancers: risks, evaluation, prevention and early detection. Obstet Gynecol Clin N Am 2007; 34: 783-802.

15. Santegoets LAM, Helmerhorst TJM, Van Der Meijden WI. A retrospective study of 95 women with a clinical diagnosis of genital lichen planus. JLGTD 2010; 14: 323-8.
16. Ording Helgesen AL, Gjersvik P, Jebsen P, et al. Vaginal involvement in genital erosive lichen planus. Acta Obstet Gynecol 2010; 89: 966-70.

17. Lazaridou E, Fotiadou C, Giannopoulou C, loannides D. Persistent erythematous lesion of the vulva: a diagnostic and treatment challenge. J Drugs Dermatol 2012; 11: 110-2.

18. van der Waal I. Potentially malignant disorders of the oral and oropharyngeal mucosa; terminology, classification and present concepts of management. Oral Oncol 2009; 45: 317-23.

19. van der Waal I. Potentially malignant disorders of the oral and oropharyngeal mucosa; present concepts of management. Oral Oncol 2010; 46: 423-5.

20. Gonzalez-Moles MA, Scully C, Gil-Montoya JA. Oral lichen planus: controversies surrounding malignant transformation. Oral Dis 2008; 14: 229-43.

21. Scully C, Carrozzo M. Oral mucosal disease: lichen planus. Brit J Oral Max Surg 2008; 46: 15-21.

22. Neppelberg E, Johannessen AC. DNA content, cyclooxygenase-2 expression and loss of E-cadherin expression do not predict risk of malignant transformation in oral lichen planus. Eur Arch Otorhinolaryngol 2007; 264: 1223-30. 